\title{
Influence of catastrophizing, anxiety, and depression on in-hospital opioid consumption, pain, and quality of recovery after adult spine surgery
}

\author{
Lauren K. Dunn, MD, PhD, ${ }^{1}$ Marcel E. Durieux, MD, PhD, ${ }^{1,2}$ Lucas G. Fernández, MD, DSc, ${ }^{1}$ \\ Siny Tsang, PhD, ${ }^{3}$ Emily E. Smith-Straesser, MD, ${ }^{1}$ Hasan F. Jhaveri, ${ }^{1}$ Shauna P. Spanos, MD, ${ }^{1}$ \\ Matthew R. Thames, MD, MBA, ${ }^{1}$ Christopher D. Spencer, MD, MSc, ${ }^{1}$ Aaron Lloyd, MD, ${ }^{1}$ \\ Russell Stuart, MD, ${ }^{1}$ Fan Ye, MD, MPH, Jacob P. Bray, MD, ${ }^{1}$ Edward C. Nemergut, MD, ${ }^{1,2}$ and \\ Bhiken I. Naik, MBBCh ${ }^{1,2}$
}

Departments of ${ }^{1}$ Anesthesiology and ${ }^{2}$ Neurosurgery, University of Virginia, Charlottesville, Virginia; and ${ }^{3}$ Department of Epidemiology, Columbia University, New York, New York

OBJECTIVE Perception of perioperative pain is influenced by various psychological factors. The aim of this study was to determine the impact of catastrophizing, anxiety, and depression on in-hospital opioid consumption, pain scores, and quality of recovery in adults who underwent spine surgery.

METHODS Patients undergoing spine surgery were enrolled in this study, and the preoperatively completed questionnaires included the verbal rating scale (VRS), Pain Catastrophizing Scale (PCS), Hospital Anxiety and Depression Scale (HADS), and Oswestry Disability Index (ODI). Quality of recovery was assessed using the 40-item Quality of Recovery questionnaire (QoR40). Opioid consumption and pain scores according to the VRS were recorded daily until discharge.

RESULTS One hundred thirty-nine patients were recruited for the study, and 101 completed the QoR40 assessment postoperatively. Patients with higher catastrophizing scores were more likely to have higher maximum pain scores postoperatively (estimate: 0.03, SE: 0.01, $p=0.02$ ), without increased opioid use (estimate: 0.44, SE: 0.27, $p=0.11$ ). Preoperative anxiety (estimate: $1.18, \mathrm{SE}: 0.65, p=0.07$ ) and depression scores (estimate: 1.06, SE: 0.71, $p=0.14$ ) did not correlate with increased postoperative opioid use; however, patients with higher preoperative depression scores had lower quality of recovery after surgery (estimate: -1.9 , SE: 0.56, $p<0.001$ ).

CONCLUSIONS Catastrophizing, anxiety, and depression play important roles in modulating postoperative pain. Preoperative evaluation of these factors, utilizing a validated tool, helps to identify patients at risk. This might allow for earlier psychological intervention that could reduce pain severity and improve the quality of recovery.

https://thejns.org/doi/abs/10.3171/2017.5.SPINE1734

KEY WORDS pain catastrophizing; anxiety; depression; spine surgery

$\mathrm{P}$ ERCEPTION and severity of perioperative pain are influenced by various biological, cultural, and psychological factors. ${ }^{31}$ The psychological factors that impact postoperative recovery include catastrophizing, anxiety, and depression. ${ }^{4}$ Catastrophizing is an exaggerated behavioral response to anticipated or actual pain and influences the perception of pain. ${ }^{10,33}$ It can account for between $7 \%$ and $13 \%$ of variation in pain scores. ${ }^{31}$ Catastrophizing includes 3 components: magnification, rumi- nation, and helplessness. ${ }^{11}$ High levels of catastrophizing are correlated with increased reported pain intensity and a greater incidence of chronic pain and are an independent determinant of disability in patients with chronic pain syndromes. ${ }^{1,8,14,20,22,32,35}$ Subsets of patients who tend to display higher levels of catastrophizing include women and younger patients. ${ }^{9} 10,12,34$

Patients with spine disease have a high incidence of chronic pain, increased disability, and poor quality of life..$^{21}$

ABBREVIATIONS ASA = American Society of Anesthesiologists; $C B T=$ cognitive behavioral therapy; HADS = Hospital Anxiety and Depression Scale; $I C U=$ intensive care unit; LMM = linear mixed effects model; ODI = Oswestry Disability Index; PACU = post-anesthesia care unit; PCA = patient-controlled analgesia; PCS = Pain Catastrophizing Scale; QoR40 = 40-item quality of recovery questionnaire; VRS = verbal rating scale.

SUBMITTED January 7, 2017. ACCEPTED May 22, 2017.

INCLUDE WHEN CITING Published online November 10, 2017; DOI: 10.3171/2017.5.SPINE1734. 
Interestingly, perceived back and leg pain and the level of disability are not correlated with objective markers of the severity of spinal disease. ${ }^{16,27}$ Psychological factors may play an important role in pain perception and the management of patients with spine-related chronic pain.,12,13

The aim of this study was to determine the impact of catastrophizing, anxiety, and depression on in-hospital opioid consumption, pain scores, and quality of recovery in adults who underwent spine surgery.

\section{Methods}

This prospective single-center observational study of adults undergoing spine surgery was approved by the University of Virginia Institutional Review Board for Health Science Research, and the need for written consent was waived. We enrolled patients between 18 and 80 years of age who were scheduled for cervical, thoracic, and/or lumbar spine surgery and could read and speak English. We excluded patients undergoing emergency surgery, pregnant females, and prisoners. Subjects were enrolled from April 2015 to April 2016.

\section{Study Protocol}

After verbal consent was obtained, demographic data were collected (patient sex, age, and American Society of Anesthesiologists [ASA] physical status classification [Arabic numbers were used for the classification to perform statistical analysis]). The preoperative use of opioid and nonopioid medications, including nonsteroidal antiinflammatory drugs, gabapentinoids, benzodiazepines, and muscle relaxants was recorded. All opioids used during the perioperative period were converted to morphine equivalents to facilitate analysis (http://www.uptodate. $\mathrm{com} /$ contents/cancerpain-management-with-opioidsoptimizing-analgesia). An 11-point verbal rating scale (VRS) was used to quantify pre- and postoperative pain.

\section{Questionnaires}

In addition to the VRS, other questionnaires completed prior to surgery included the 1) Pain Catastrophizing Scale (PCS), a 13-item scale that evaluates rumination, magnification, and helplessness associated with pain, with each item rated on a 5-point scale: 0 (not at all) to 4 (all the time); 2) Hospital Anxiety and Depression Scale (HADS), a 14-item scale (7 items each for anxiety and depression), with each item scored from 0 to 3; and 3) Oswestry Disability Index (ODI). ${ }^{6,11,27}$

Quality of recovery after surgery was assessed using the 40-item quality of recovery questionnaire (QoR40), which measures 5 aspects (dimensions) of recovery: emotional state (9 items), physical comfort (12 items), psychological support (7 items), physical independence (5 items), and pain (7 items). A composite score for the QoR40 was used for analysis in this study. ${ }^{17}$

\section{Intraoperative and Postoperative Data}

Collected intraoperative data included duration of procedure, time to extubation, and total administration of intraoperative opioid and nonopioid analgesics (lidocaine, ketamine, dexmedetomidine, intravenous acetaminophen). Surgery-specific data included primary versus repeat surgery, site of surgery (cervical, thoracic, or lumbar), anterior versus posterior approach, number of intervertebral procedures (transforaminal lumbar interbody fusions), and number of osteotomies performed.

Collected postoperative data included total daily opioid consumption, nonopioid medications (including lidocaine, ketamine, dexmedetomidine, and acetaminophen), daily VRS score (including the highest, lowest, and median scores), time to first ambulation, intensive care unit (ICU) length of stay, and hospital length of stay.

\section{Perioperative Analgesia}

Postoperatively, all study participants were admitted to either the post-anesthesia care unit (PACU) or the ICU based on the extent of surgery and consistent with our institutional practice. Postoperative opioid analgesia was administered according to a standardized protocol. This included the use of a hydromorphone patient-controlled analgesia (PCA) pump with a patient-controlled bolus dose of $0.1 \mathrm{mg}$ with a lockout period of 8 minutes. If pain control was inadequate, the PCA pump could be adjusted according to standardized institutional practice. In addition to the intravenous opioid regimen, oral oxycodone was initiated when oral intake was commenced and was increased if pain control remained inadequate.

\section{Statistical Analysis}

Continuous variables are presented as the mean and standard deviation. Dichotomous variables are presented as percentages, whereas count variables are presented as the median and range. Linear regression models were used to examine the associations between preoperative and postoperative characteristics.

The effects of preoperative characteristics (catastrophizing, anxiety, and depression) on repeatedly measured postoperative characteristics were examined using mixed effects models, controlling for patient demographic characteristics, preoperative morphine use, surgical procedures, and intraoperative characteristics. Linear mixed effects models (LMMs) were used for continuous postoperative characteristics (opioid use and pain scores). The LMMs are adopted to take into account the correlated observations within subjects. For each observation $j$ in patient $i$, the general form of the LMM is written as follows: $y_{i j}=\beta_{0}+\beta_{1} x_{l i j}+\beta_{2} x_{2 i j} \ldots \beta_{n} x_{n i j}+b_{i 0}+b_{i l} z_{l i j}+\ldots b_{i n} z_{n i j}+$ $\varepsilon_{i j}$, where $y_{i j}$ is the outcome variable, $\beta_{n}$ are the fixed effect coefficients associated with the fixed effect predictors $x_{n i j}$, $b_{i n}$ are the random effect coefficients associated with the random effect predictors $z_{n i j}$, and $\varepsilon_{i j} i$ s the random error.

In this study, the preoperative characteristic of interest (catastrophizing [PCS score], anxiety [HADS score], or depression [HADS score]) and time (that is, number of days postoperatively) were modeled as fixed effects $x_{i j}$ and $x_{2 i j}$, respectively. To take into account patient demographic characteristics, preoperative morphine use, surgical procedures, and intraoperative characteristics, these variables were each modeled as fixed effects $\left(x_{n i j}, \ldots, x_{n i j}\right)$. Two random effects were included: the random intercept $\left(b_{i 0}\right)$ 
TABLE 1. Demographics and preoperative analgesic use in 138 patients undergoing spine surgery

\begin{tabular}{lcccc}
\hline \multicolumn{1}{c}{ Parameter } & All & Men & Women & $\begin{array}{c}\text { P } \\
\text { Value }\end{array}$ \\
\hline No. of patients & 138 & 81 & 57 & \\
\hline Mean age in yrs & $61(11)$ & $61(10)$ & $61(12)$ & 0.86 \\
\hline Mean BMI in kg/m² & $32(7)$ & $32(6)$ & $31(9)$ & 0.88 \\
\hline Mean ASA physical status & $2.5(0.6)$ & $2.5(0.6)$ & $2.5(0.5)$ & 0.53 \\
\hline Mean VRS score & $6(3)$ & $6(3)$ & $6(3)$ & 0.66 \\
\hline Mean PCS score & $20(11)$ & $21(11)$ & $19(10)$ & 0.29 \\
\hline Mean ODI & $47(17)$ & $46(18)$ & $48(15)$ & 0.51 \\
\hline Mean anxiety HADS score & $8(5)$ & $8(4)$ & $8(5)$ & 0.71 \\
\hline $\begin{array}{l}\text { Mean depression HADS } \\
\text { score }\end{array}$ & $7(4)$ & $7(4)$ & $7(4)$ & 0.72 \\
\hline $\begin{array}{l}\text { Mean preop opioid: mor- } \\
\text { phine equivalent dose } \\
\text { in mg }\end{array}$ & $11(14)$ & $10(14)$ & $11(14)$ & 0.63 \\
\hline \% taking NSAID & & & & \\
\hline \% taking benzodiazepine & 20 & 17 & 25 & 0.41 \\
\hline \% taking muscle relaxant & 27 & 28 & 25 & 0.76 \\
\hline \% taking tramadol & 28 & 26 & 30 & 0.76 \\
\hline
\end{tabular}

$\mathrm{BMI}=$ body mass index; NSAID = nonsteroidal antiinflammatory drug. Data presented as the mean (standard deviation) or percentage. Sex differences were examined using linear regression models (for continuous variables), and chi-square statistics (for dichotomous variables).

* Arabic numerals were used for the classification to perform statistical analysis.

and the random slope $\left(b_{i l}\right)$ of time (that is, number of days postoperatively) for patient $i$.

Strength of correlation was interpreted as follows:
$0.00-0.19$, very weak; $0.20-0.39$, weak; $0.40-0.59$, moderate; $0.60-0.79$, strong; and $0.80-1.0$, very strong.

All statistical analyses were conducted using the statistical program R 3.2.3 (R Foundation). Linear mixed effects models were performed using the "Ime4" package in $\mathrm{R}^{3}{ }^{3}$

\section{Results}

A total of 139 patients were recruited, and 1 of these patients was excluded because of significant daily opioid use from an opioid intrathecal pump. One hundred one patients completed the QoR40 assessment postoperatively. Demographic and preoperative psychosocial characteristics are presented in Table 1. No significant differences were noted between men and women with regard to demographics or preoperative opioid and nonopioid medication use.

Patients with PCS scores $\geq 30$ were classified as having pain catastrophizing. ${ }^{30}$ Most patients $(107[79.2 \%])$ had a PCS score < 30, whereas $28(20.7 \%)$ had a PCS score $\geq 30$; 3 patients did not complete the PCS questionnaire. Patient scores on the HADS can be categorized as normal (0-7), borderline case (8-10), and abnormal case (11-21) for depression and anxiety. Eighty-four patients $(61.3 \%)$ were normal, 27 (19.7\%) were borderline cases, and 26 (19.0\%) were abnormal cases for depression (1 patient did not complete the HADS). Seventy-one patients $(51.8 \%)$ were normal, 27 (19.7\%) were borderline cases, and 39 (28.5\%) were abnormal cases for anxiety.

Opioid and nonopioid use and other surgical data are listed in Table 2. Of note, male patients had longer operations (male: $316 \pm 126$ minutes vs female: $248 \pm 97$ minutes, $\mathrm{p}<0.05$ ) and a higher total intraoperative ket-

TABLE 2. Intraoperative and postoperative data

\begin{tabular}{|c|c|c|c|c|}
\hline Parameter & All & Men & Women & $\mathrm{p}$ Value \\
\hline \multicolumn{5}{|l|}{ Intraop } \\
\hline Mean total opioid dose in mg & $20(26)$ & $22(33)$ & $16(10)$ & 0.15 \\
\hline Mean lidocaine dose in mg & $790(631)$ & $844(487)$ & $712(791)$ & 0.23 \\
\hline Mean Dex dose in $\mu \mathrm{g}$ & $9(41)$ & $9(40)$ & $9(40)$ & 0.98 \\
\hline Mean ketamine dose in mg & $71(83)$ & $85(96)$ & $51(56)$ & 0.02 \\
\hline Mean acetaminophen dose in $\mathrm{mg}$ & $359(508)$ & $321(520)$ & $412(490)$ & 0.3 \\
\hline Mean operation time in mins & $288(119)$ & $316(126)$ & $248(97)$ & $<0.01$ \\
\hline$\%$ undergoing redo surgery & 49 & 52 & 46 & 0.58 \\
\hline$\%$ undergoing pst approach & 86 & 86 & 84 & 0.91 \\
\hline$\%$ undergoing lumbar surgery & 83 & 83 & 82 & 1.00 \\
\hline Median no. of osteotomies (range) & $0(0-8)$ & $0(0-8)$ & $0(0-6)$ & 0.07 \\
\hline Median no. of TLIFs (range) & $1(0-3)$ & $1(0-3)$ & $0(0-3)$ & 0.42 \\
\hline \multicolumn{5}{|l|}{ Postop } \\
\hline Mean QoR40 score & $152(21)$ & $151(22)$ & $154(20)$ & 0.60 \\
\hline Mean time to ambulation in days & $2(1)$ & $2(2)$ & $2(1)$ & 0.89 \\
\hline Mean ICU LOS in hrs & $22(26)$ & $21(23)$ & $25(30)$ & 0.37 \\
\hline Mean hospital LOS in days & $5(3)$ & $5(3)$ & $5(3)$ & 0.79 \\
\hline
\end{tabular}

Dex = dexmedetomidine; $\mathrm{LOS}=$ length of stay; pst = posterior; TLIF = transforaminal lumbar interbody fusion.

Data presented as the mean (standard deviation), percentage, or median (range), as appropriate. Sex differences were examined using linear regression models (for continuous variables), chi-square statistics (for dichotomous variables), and generalized linear regression models (for count variables). 
TABLE 3. Linear mixed effects models estimating the effect of preoperative pain catastrophizing, depression, and anxiety on postoperative opioid use*

\begin{tabular}{|c|c|c|c|c|c|c|c|c|c|c|c|}
\hline Catastrophizing Model & Estimate & SE & $\begin{array}{c}\mathrm{p} \\
\text { Value }\end{array}$ & Depression Model & Estimate & SE & $\begin{array}{c}\mathrm{p} \\
\text { Value }\end{array}$ & Anxiety Model & Estimate & SE & $\begin{array}{c}p \\
\text { Value }\end{array}$ \\
\hline \multicolumn{12}{|l|}{ Fixed effect } \\
\hline Intercept & 99.8 & 50.3 & 0.05 & Intercept & 111.5 & 48.4 & 0.02 & Intercept & 97.1 & 49.6 & 0.05 \\
\hline PCS score & 0.4 & 0.3 & 0.11 & $\begin{array}{l}\text { Depression HADS } \\
\text { score }\end{array}$ & 1.1 & 0.7 & 0.14 & $\begin{array}{l}\text { Anxiety HADS } \\
\text { score }\end{array}$ & 1.2 & 0.7 & 0.07 \\
\hline Time (days) & -6.6 & 0.8 & $<0.001$ & Time (days) & -6.7 & 0.8 & $<0.001$ & Time (days) & -6.7 & 0.8 & $<0.001$ \\
\hline Age & -0.6 & 0.3 & 0.04 & Age & -0.7 & 0.3 & 0.01 & Age & -0.6 & 0.3 & 0.06 \\
\hline Sex (female) & -5.6 & 5.5 & 0.31 & Sex (female) & -7.3 & 5.4 & 0.18 & Sex (female) & -7.4 & 5.3 & 0.17 \\
\hline $\log (\mathrm{BMI})$ & -13.1 & 13.6 & 0.34 & $\log (\mathrm{BMI})$ & -16.1 & 13.4 & 0.23 & $\log (\mathrm{BMI})$ & -15.2 & 13.3 & 0.25 \\
\hline Preop morphine use & 0.9 & 0.2 & $<0.001$ & Preop morphine use & 0.9 & 0.2 & $<0.001$ & Preop morphine use & 0.9 & 0.2 & $<0.001$ \\
\hline Procedure (redo) & 14.0 & 5.5 & 0.01 & Procedure (redo) & 12.2 & 5.4 & 0.02 & Procedure (redo) & 13.1 & 5.4 & 0.02 \\
\hline Approach (pst) & 5.3 & 15.0 & 0.72 & Approach (pst) & 6.3 & 14.8 & 0.67 & Approach (pst) & 6.4 & 14.7 & 0.66 \\
\hline No. of osteotomies & 3.3 & 1.7 & 0.05 & No. of osteotomies & 3.6 & 1.7 & 0.03 & No. of osteotomies & 3.4 & 1.6 & 0.04 \\
\hline TLIF & 5.0 & 3.4 & 0.14 & TLIF & 3.6 & 3.3 & 0.28 & TLIF & 3.9 & 3.3 & 0.24 \\
\hline $\begin{array}{l}\text { Surgical site } \\
\text { (lumbar) }\end{array}$ & 5.1 & 13.9 & 0.71 & $\begin{array}{l}\text { Surgical site } \\
\text { (lumbar) }\end{array}$ & 8.7 & 13.6 & 0.52 & $\begin{array}{l}\text { Surgical site } \\
\text { (lumbar) }\end{array}$ & 8.9 & 13.5 & 0.51 \\
\hline Intraop opioids & 0.1 & 0.10 & 0.10 & Intraop opioids & 0.2 & 0.10 & 0.09 & Intraop opioids & 0.1 & 0.1 & 0.14 \\
\hline Lidocaine & 0.0 & 0.0 & 0.58 & Lidocaine & 0.0 & 0.00 & 0.63 & Lidocaine & 0.0 & 0.00 & 0.68 \\
\hline Dex & 0.1 & 0.07 & 0.12 & Dex & 0.1 & 0.07 & 0.15 & Dex & 0.09 & 0.07 & 0.16 \\
\hline Ketamine & 0.04 & 0.04 & 0.38 & Ketamine & 0.02 & 0.04 & 0.52 & Ketamine & 0.02 & 0.04 & 0.54 \\
\hline Acetaminophen & -0.0 & 0.01 & 0.86 & Acetaminophen & -0.0 & 0.01 & 0.96 & Acetaminophen & 0.0 & 0.01 & 0.93 \\
\hline Operation length & -0.04 & 0.03 & 0.29 & Operation length & -0.03 & 0.03 & 0.39 & Operation length & -0.03 & 0.03 & 0.45 \\
\hline $\begin{array}{l}\text { Extubation in OR } \\
\text { (yes) }\end{array}$ & -1.9 & 8.5 & 0.82 & $\begin{array}{l}\text { Extubation in OR } \\
\text { (yes) }\end{array}$ & 3.3 & 8.3 & 0.70 & $\begin{array}{l}\text { Extubation in OR } \\
\text { (yes) }\end{array}$ & 3.1 & 8.2 & 0.71 \\
\hline \multicolumn{12}{|l|}{ Random effect } \\
\hline Intercept & 1165 & & & & 1103 & & & & 3.47 & & \\
\hline Time (days) & 41 & & & & 41 & & & & 0.13 & & \\
\hline
\end{tabular}

OR = operating room; SE = standard error; Time (days) = number of days postoperatively.

* Controlling for patient demographics, preoperative morphine use, surgical procedures, and intraoperative variables.

amine dose (male: $85 \pm 96 \mathrm{mg}$ vs female: $51 \pm 56 \mathrm{mg}, \mathrm{p}=$ 0.02). Postoperative ICU time, hospital length of stay, and QoR40 scores were not significantly different between men and women.

\section{Opioid Consumption}

The effects of preoperative catastrophizing, depression, and anxiety on in-hospital postoperative opioid consumption were examined using LMMs, after taking into account patient age, sex, preoperative opioid consumption, and characteristics of surgical procedures. Preoperative catastrophizing (estimate: 0.44 , SE: $0.27, \mathrm{p}=0.11$ ), depression (estimate: $1.06, \mathrm{SE}: 0.71, \mathrm{p}=0.14$ ), and anxiety (estimate: 1.18 , SE: $0.65, \mathrm{p}=0.07)$ were not associated with increased postoperative opioid use (Table 3). After controlling for demographic, surgical, and psychological factors, we found that preoperative opioid use was associated with increased postoperative opioid consumption.

\section{Pain Scores}

The effects of preoperative catastrophizing on postoperative pain scores were examined using LMMs, after taking into account patient age, sex, preoperative opioid consumption, and characteristics of surgical procedures. Preoperative catastrophizing was associated with the maximum (estimate: 0.03 , SE: $0.01, p=0.02$ ) and median (estimate: 0.03 , SE: $0.02, p=0.03$ ) VRS scores, suggesting that patients with increased catastrophizing were more likely to have higher postoperative maximum and median VRS scores than those with lower catastrophizing scores (Table 4). The effect can be interpreted, holding other variables constant, as follows: a 1-unit increase in the PCS is associated with a 0.03 -unit increase in the maximum postoperative VRS score. In contrast, catastrophizing was not associated with postoperative minimum VRS scores (estimate: 0.0, SE: 0.02, p = 0.89). Of note, the maximum, minimum, and median VRS scores were significantly correlated with age, with older patients reporting lower VRS scores than the younger patients.

\section{Postoperative Quality of Recovery}

There was a statistically significant negative effect of intraoperative opiates on QoR40, indicating that patients who had received more intraoperative opiates reported lower QoR40 scores postoperatively than those who had received less intraoperative opiates (Table 5). The effect of 
TABLE 4. Linear mixed effects model estimating the effect of preoperative pain catastrophizing on postoperative median, maximum, and minimum VRS scores*

\begin{tabular}{|c|c|c|c|c|c|c|c|c|c|}
\hline \multirow[b]{2}{*}{ Model } & \multicolumn{3}{|c|}{ Median VRS } & \multicolumn{3}{|c|}{ Maximum VRS } & \multicolumn{3}{|c|}{ Minimum VRS } \\
\hline & Estimate & SE & $p$ Value & Estimate & SE & $p$ Value & Estimate & SE & $p$ Value \\
\hline \multicolumn{10}{|l|}{ Fixed effect } \\
\hline Intercept & 11.1 & 3 & $<0.001$ & 9.7 & 2.6 & $<0.001$ & 9.1 & 3 & $<0.001$ \\
\hline PCS score & 0.03 & 0.02 & 0.03 & 0.03 & 0.01 & 0.02 & -0.0 & 0.02 & 0.89 \\
\hline Time (days) & -0.19 & 0.05 & $<0.001$ & -0.30 & 0.04 & $<0.001$ & 0.0 & 0.04 & 0.97 \\
\hline Age & -0.05 & 0.02 & $<0.001$ & -0.03 & 0.01 & 0.02 & -0.05 & 0.02 & $<0.001$ \\
\hline Sex (female) & 0.2 & 0.3 & 0.53 & 0.1 & 0.3 & 0.62 & -0.2 & 0.3 & 0.53 \\
\hline $\log (\mathrm{BMI})$ & -0.9 & 0.8 & 0.27 & -0.1 & 0.7 & 0.84 & -0.9 & 0.8 & 0.25 \\
\hline Preop morphine & 0.02 & 0.01 & 0.16 & 0.01 & 0.01 & 0.25 & 0.04 & 0.01 & $<0.001$ \\
\hline Procedure (redo) & 0.5 & 0.3 & 0.12 & 0.2 & 0.3 & 0.55 & 0.4 & 0.3 & 0.28 \\
\hline Approach (pst) & -0.7 & 0.9 & 0.44 & 0.2 & 0.8 & 0.83 & -0.2 & 0.9 & 0.84 \\
\hline No. of osteotomies & 0.1 & 0.1 & 0.21 & 0.2 & 0.09 & 0.07 & 0.2 & 0.1 & 0.05 \\
\hline TLIF & 0.2 & 0.2 & 0.24 & 0.3 & 0.2 & 0.12 & 0.2 & 0.2 & 0.46 \\
\hline Surgical site (lumbar) & 0.6 & 0.8 & 0.49 & 0.4 & 0.7 & 0.55 & -0.05 & 0.8 & 0.95 \\
\hline Total opiates & 0.0 & 0.01 & 0.41 & 0.01 & 0.01 & 0.08 & -0.01 & 0.01 & 0.09 \\
\hline Lidocaine & 0.0 & 0.0 & 0.60 & 0.0 & 0.0 & 0.27 & -0.0 & 0.00 & 0.75 \\
\hline Dex & 0.01 & 0.0 & 0.13 & 0.01 & 0.0 & 0.04 & 0.01 & 0.00 & 0.02 \\
\hline Ketamine & -0.0 & 0.0 & 0.75 & -0.0 & 0.0 & 0.26 & -0.0 & 0.00 & 0.99 \\
\hline Acetaminophen & -0.0 & 0.0 & 0.38 & -0.0 & 0.0 & 0.55 & -0.0 & 0.00 & 0.10 \\
\hline Operation length & -0.0 & 0.0 & 0.22 & -0.0 & 0.0 & 0.18 & -0.0 & 0.00 & 0.28 \\
\hline Extubation in OR (yes) & 0.17 & 0.5 & 0.74 & 0.2 & 0.4 & 0.65 & 0.09 & 0.5 & 0.85 \\
\hline \multicolumn{10}{|l|}{ Random effect } \\
\hline Intercept & 3.5 & & & 2.3 & & & 2.10 & & \\
\hline Time (days) & 0.1 & & & 0.08 & & & 3.11 & & \\
\hline
\end{tabular}

* Controlling for patient demographics, preoperative morphine use, surgical procedures, and intraoperative variables.

preoperative catastrophizing, depression, and anxiety on quality of recovery was examined using multiple regression models, controlling for patient age, sex, preoperative opioid use, and characteristics of surgical procedures. Results showed no statistically significant effects of catastrophizing (estimate: -0.13 , SE: $0.23, p=0.56$ ) or anxiety (estimate: -0.56 , SE: $0.55, \mathrm{p}=0.31$ ) on QoR40 scores; however, depression was found to be negatively associated with QoR40 scores (estimate: -1.9 , SE: $0.56, \mathrm{p}<0.001$ ). The effect of depression on the QoR40 can be interpreted, holding other variables constant, as follows: a 1-unit increase in the HADS depression score is associated with 1.9-unit decrease in the QoR40 score. This suggests that patients who are more depressed preoperatively are more likely to report lower quality of recovery scores after surgery.

\section{Correlations}

We examined correlations between patient age and preoperative characteristics including the pain score, PCS score, ODI, HADS depression score, HADS anxiety score, and the amount of preoperative opioid use (morphineequivalent dose; Table 6). Patient age was very weakly correlated with ODI $(r=-0.19, p=0.02)$; weakly correlated with preoperative VRS score $(\mathrm{r}=-0.2, \mathrm{p}=0.02)$, PCS $(\mathrm{r}$ $=-0.3, \mathrm{p}<0.001)$, preoperative opioid use $(\mathrm{r}=-0.23, \mathrm{p}=$ $0.007)$, and depression $(r=-0.29, p<0.001)$; and moder- ately correlated with anxiety $(\mathrm{r}=-0.51, \mathrm{p}<0.001)$. Age was weakly correlated with ASA status $(r=0.22, p=0.01)$, reflective of increasing comorbidity with age.

Preoperative VRS score was weakly correlated with PCS $(r=0.39, p<0.001)$, depression $(r=0.30, p<0.001)$, and anxiety $(\mathrm{r}=0.33, \mathrm{p}<0.001)$ and moderately correlated with ODI $(\mathrm{r}=0.51, \mathrm{p}<0.001)$.

Preoperative opioid consumption was very weakly correlated with preoperative VRS score $(r=0.19, p=0.03)$ and depression $(\mathrm{r}=0.18, \mathrm{p}=0.04)$ but not with PCS ( $\mathrm{r}=$ $0.16, p=0.07)$ or anxiety $(r=0.16, p=0.07)$.

Finally, preoperative variables that were correlated with postoperative variables included time to ambulation, hospital and ICU length of stay, and the QoR40 (Table 7). Age was weakly correlated with ICU stay $(r=0.24, p<0.01)$ and hospital stay $(r=0.21, p<0.05)$ but no correlation was found between age and QoR40 postoperatively $(r=0.02)$.

\section{Discussion}

This study demonstrated that after controlling for age, sex, preoperative opioid use, and characteristics of surgical procedures, there was no correlation between higher catastrophizing scores and increased in-hospital opioid use or a poorer quality of recovery. However, patients with higher catastrophizing scores were more likely to have higher maximum pain scores postoperatively. In contrast to previ- 
TABLE 5. Multiple regression models estimating the effect of pain catastrophizing, depression, and anxiety on postoperative QoR40 score*

\begin{tabular}{|c|c|c|c|c|c|c|c|c|c|c|c|}
\hline Fixed Effect & Estimate & SE & $\begin{array}{c}p \\
\text { Value }\end{array}$ & Fixed Effect & Estimate & SE & $\begin{array}{c}\mathrm{p} \\
\text { Value }\end{array}$ & Fixed Effect & Estimate & SE & $\begin{array}{c}\mathrm{p} \\
\text { Value }\end{array}$ \\
\hline Intercept & 114.1 & 42.5 & 0.01 & Intercept & 109.6 & 36.5 & $<0.001$ & Intercept & 106.9 & 39.6 & 0.01 \\
\hline PCS score & -0.1 & 0.2 & 0.56 & $\begin{array}{l}\text { Depression HADS } \\
\text { score }\end{array}$ & -1.9 & 0.6 & $<0.001$ & Anxiety HADS score & -0.6 & 0.6 & 0.31 \\
\hline Age & -0.05 & 0.2 & 0.84 & Age & -0.1 & 0.2 & 0.72 & Age & -0.03 & 0.2 & 0.89 \\
\hline Sex (female) & 3.7 & 5.1 & 0.47 & Sex (female) & 5.8 & 4.4 & 0.20 & Sex (female) & 5.0 & 4.7 & 0.29 \\
\hline $\log (\mathrm{BMI})$ & 9.8 & 11.9 & 0.41 & $\log (\mathrm{BMI})$ & 17.6 & 10.6 & 0.10 & $\log (\mathrm{BMI})$ & 14.4 & 11.2 & 0.20 \\
\hline Preop morphine & -0.1 & 0.2 & 0.69 & Preop morphine & 0.01 & 0.2 & 0.96 & Preop morphine & -0.1 & 0.2 & 0.65 \\
\hline Procedure (redo) & 1.7 & 5 & 0.74 & Procedure (redo) & 2.3 & 4.4 & 0.61 & Procedure (redo) & 1.3 & 4.7 & 0.78 \\
\hline Approach (pst) & 2.3 & 12.8 & 0.85 & Approach (pst) & 2.7 & 11.4 & 0.81 & Approach (pst) & 1.9 & 12.1 & 0.87 \\
\hline No. of osteotomies & 0.6 & 1.5 & 0.67 & No. of osteotomies & 0.5 & 1.3 & 0.73 & No. of osteotomies & 0.6 & 1.4 & 0.66 \\
\hline TLIF & 0.5 & 3.1 & 0.86 & TLIF & 1.7 & 2.7 & 0.53 & TLIF & 1.5 & 2.9 & 0.60 \\
\hline Surgical site (lumbar) & -9.8 & 12 & 0.41 & Surgical site (lumbar) & -12.5 & 10.6 & 0.24 & Surgical site (lumbar) & -11.4 & 11.3 & 0.31 \\
\hline Intraop opioids & -0.2 & 0.1 & 0.03 & Intraop opioids & -0.2 & 0.1 & 0.01 & Intraop opioids & -0.2 & 0.1 & 0.04 \\
\hline Lidocaine & -0.0 & 0.0 & 0.65 & Lidocaine & -0.0 & 0.00 & 0.36 & Lidocaine & -0.0 & 0.00 & 0.65 \\
\hline Dex & -0.02 & 0.1 & 0.71 & Dex & -0.04 & 0.1 & 0.51 & Dex & -0.02 & 0.1 & 0.72 \\
\hline Ketamine & 0.02 & 0.04 & 0.70 & Ketamine & 0.01 & 0.03 & 0.80 & Ketamine & 0.03 & 0.04 & 0.47 \\
\hline Acetaminophen & 0.0 & 0.0 & 0.62 & Acetaminophen & -0.0 & 0.00 & 0.88 & Acetaminophen & -0.00 & 0.00 & 0.93 \\
\hline Operation length & 0.04 & 0.03 & 0.29 & Operation length & 0.03 & 0.03 & 0.37 & Operation length & 0.02 & 0.03 & 0.45 \\
\hline $\begin{array}{l}\text { Extubation in OR } \\
\text { (yes) }\end{array}$ & 6.9 & 8.7 & 0.43 & $\begin{array}{l}\text { Extubation in OR } \\
\text { (yes) }\end{array}$ & 0.3 & 7.62 & 0.97 & $\begin{array}{l}\text { Extubation in OR } \\
\text { (yes) }\end{array}$ & 3 & 8.0 & 0.71 \\
\hline
\end{tabular}

${ }^{*}$ Controlling for patient demographics, preoperative morphine use, surgical procedures, and intraoperative variables.

ously published results, we found that preoperative anxiety and depression scores were not correlated with increased in-hospital opioid use; however, patients with higher preoperative depression scores were more likely to report a poorer quality of recovery. Finally, we demonstrated that preoperative pain scores, catastrophizing, depression, anxiety, and disability were all positively correlated with one another.

Our results are consistent with previously published studies in both Southern ${ }^{19}$ and Northern ${ }^{2}$ European patients. In 61 Greek patients undergoing 1-level posterior lumbar spine fusion, Papaioannou et al. reported that catastrophizing predicted postoperative pain intensity and analgesic use. ${ }^{19}$ A limitation of their study was that preoperative analgesic use and pain scores were not reported. Among Swedish patients undergoing lumbar spine fusion, Abbott et al. found that preoperative catastrophizing predicted back pain intensity and functional disability up to

TABLE 6. Correlation coefficients of preoperative variables

\begin{tabular}{|c|c|c|c|c|c|c|c|c|}
\hline \multirow[b]{2}{*}{ Preop Variable } & \multicolumn{8}{|c|}{ Preop Variable } \\
\hline & Age & $\mathrm{BMI}$ & ASA Status & VRS Score & PCS Score & ODI & $\begin{array}{l}\text { Depression HADS } \\
\text { Score }\end{array}$ & $\begin{array}{c}\text { Anxiety HADS } \\
\text { Score }\end{array}$ \\
\hline \multicolumn{9}{|l|}{ Age } \\
\hline BMI & -0.05 & & & & & & & \\
\hline ASA status & $0.22^{*}$ & $0.22^{*}$ & & & & & & \\
\hline VRS score & $-0.20^{*}$ & -0.08 & 0.09 & & & & & \\
\hline PCS score & $-0.30 \dagger$ & -0.06 & 0.01 & $0.39 \dagger$ & & & & \\
\hline ODI & $-0.19^{*}$ & -0.04 & 0.02 & $0.51 \dagger$ & $0.52 \dagger$ & & & \\
\hline $\begin{array}{l}\text { Depression HADS } \\
\text { score }\end{array}$ & $-0.29 \dagger$ & 0.04 & 0.09 & $0.30 \dagger$ & $0.51 \dagger$ & $0.57 \dagger$ & & \\
\hline $\begin{array}{l}\text { Anxiety HADS } \\
\text { score }\end{array}$ & $-0.51 \dagger$ & -0.02 & -0.05 & $0.33 \dagger$ & $0.64 \dagger$ & $0.41 \dagger$ & $0.65 \dagger$ & \\
\hline Morphine use & $-0.23 \ddagger$ & $0.26 \ddagger$ & 0.08 & $0.19^{*}$ & 0.16 & $0.18^{*}$ & $0.18^{*}$ & 0.16 \\
\hline
\end{tabular}


TABLE 7. Correlation coefficients of postoperative variables

\begin{tabular}{|c|c|c|c|c|c|c|}
\hline Parameter & Preop Age & Preop BMI & Preop ASA Status & Postop Ambulation & Postop ICU LOS & Postop Hospital LOS \\
\hline \multicolumn{7}{|l|}{ Preop age } \\
\hline Preop BMI & -0.05 & & & & & \\
\hline Preop ASA status & $0.22^{*}$ & $0.22^{*}$ & & & & \\
\hline Postop ambulation & 0.05 & -0.07 & 0.03 & & & \\
\hline Postop ICU LOS & $0.24 \dagger$ & -0.02 & 0.05 & $0.34 \ddagger$ & & \\
\hline Postop hospital LOS & $0.21^{*}$ & -0.04 & $0.18^{*}$ & $0.50 \ddagger$ & $0.55 \ddagger$ & \\
\hline Postop QoR40 & 0.02 & 0.12 & -0.13 & -0.10 & -0.05 & -0.16 \\
\hline
\end{tabular}

2-3 years after surgery. ${ }^{2}$ However, cultural influences play an important role in pain perception, ${ }^{23,24}$ and these findings may not be applicable to a North American population.

Approximately $68 \%-71 \%$ of patients presenting for spine surgery use opioids preoperatively, which reflects the high prevalence of chronic pain in this population. ${ }^{16,18,27}$ Identification and treatment of high-risk patients via psychological screening prior to surgery may provide benefit for postoperative recovery. Recently, Caumo et al. developed a brief measure of emotional preoperative stress (BMEPS) to aid clinicians in identifying patients at risk for developing moderate to severe pain postoperatively. ${ }^{5}$ The B-MEPS index, which was developed utilizing previously validated measures to assess psychological factors preoperatively, is simplified to 15 questions. Although not validated in patients with spine disease, the B-MEPS offers a simple, integrated tool that identifies patients at risk for moderate to severe pain postoperatively.

Interventions that can be initiated preoperatively might include cognitive behavioral therapy (CBT) and distraction techniques. The two components of CBT aim to modify dysfunctional thought and change behavior. In nonoperative chronic low-back pain patients, van Tulder et al. reported that behavioral therapy had a moderate positive effect on pain intensity, as well as small positive effects on generic functional status and behavioral outcomes. ${ }^{36}$ The distraction technique, whereby patients focus on topics unrelated to their pain, is another tool available for the high-risk patient. Schreiber et al. reported that distraction techniques appear to be most beneficial in patients with chronic pain who are high catastrophizers, similar to the population described in our study. ${ }^{26}$ A customized enhanced recovery pathway for high-risk patients can include preoperative CBT, distraction, education, and the increased use of nonopioid adjuncts (ketamine and lidocaine) to reduce the risk of severe postoperative pain.,15

According to our study, an enhanced recovery program for spine surgery should be customized based on age as older patients report lower catastrophizing scores. The influence of age on catastrophizing is similar to that observed for other surgical procedures such as head and neck and breast surgery. ${ }^{9,29}$ An important difference is that catastrophizing in younger patients is associated with emotional responses, while in older patients it is associated with pain intensity scale and thus requires different behavioral modification interventions. ${ }^{25}$ This is relevant for spine surgery as adolescent idiopathic scoliosis and degenerative spine disease have a typical bimodal age distribution.

There are several limitations to our study. First, it was a single-center study in a North American population undergoing spine surgery. As mentioned above, our findings must be interpreted with caution in other cultural groups and in patients undergoing nonspine surgery. Second, only $73 \%$ of patients completed the QoR40 prior to discharge. This may have affected our findings about the preoperative psychological influences on postoperative quality of recovery. Third, long-term clinical outcomes related to catastrophizing, anxiety, or depression were not assessed in our study. Future studies with 1- and 2-year follow-ups will be important to determine long-term recovery and the risk of developing chronic pain related to preoperative psychological factors. A final limitation of our study was that QoR40 scores were not adjusted for perioperative clinical events and outcomes.

\section{Conclusions}

Pain catastrophizing and depression play important roles in both the perception of postoperative pain and the subjective assessment of the quality of postoperative recovery, respectively. Identifying these factors preoperatively, utilizing a validated tool, allows for earlier psychological intervention that could reduce pain severity and improve the quality of recovery.

\section{References}

1. Abbott AD, Tyni-Lenné R, Hedlund R: The influence of psychological factors on pre-operative levels of pain intensity, disability and health-related quality of life in lumbar spinal fusion surgery patients. Physiotherapy 96:213-221, 2010

2. Abbott AD, Tyni-Lenné R, Hedlund R: Leg pain and psychological variables predict outcome 2-3 years after lumbar fusion surgery. Eur Spine J 20:1626-1634, 2011

3. Bates D, Mächler M, Bolker B, Walker S: Fitting linear mixed-effects models using lme4. J Stat Softw 67:101, 2015

4. Boutin RD, Williamson MR: Magnetic resonance imaging of musculoskeletal tumors. West J Med 156:295, 1992

5. Caumo W, Nazare Furtado da Cunha M, Camey S, Maris de Jezus Castro S, Torres IL, Cadore Stefani L: Development, psychometric evaluation and validation of a brief measure of emotional preoperative stress (B-MEPS) to predict moderate to intense postoperative acute pain. Br J Anaesth 117:642649,2016 
6. Fairbank JC, Pynsent PB: The Oswestry Disability Index. Spine (Phila Pa 1976) 25:2940-2952, 2000

7. Farag E, Ghobrial M, Sessler DI, Dalton JE, Liu J, Lee JH, et al: Effect of perioperative intravenous lidocaine administration on pain, opioid consumption, and quality of life after complex spine surgery. Anesthesiology 119:932-940, 2013

8. Haythornthwaite JA, Benrud-Larson LM: Psychological aspects of neuropathic pain. Clin J Pain 16 (2 Suppl):S101S105, 2000

9. Jacobsen PB, Butler RW: Relation of cognitive coping and catastrophizing to acute pain and analgesic use following breast cancer surgery. J Behav Med 19:17-29, 1996

10. Keefe FJ, Rumble ME, Scipio CD, Giordano LA, Perri LM: Psychological aspects of persistent pain: current state of the science. J Pain 5:195-211, 2004

11. Khan RS, Ahmed K, Blakeway E, Skapinakis P, Nihoyannopoulos L, Macleod K, et al: Catastrophizing: a predictive factor for postoperative pain. Am J Surg 201:122-131, 2011

12. Kim HJ, Cho CH, Kang KT, Chang BS, Lee CK, Yeom JS: The significance of pain catastrophizing in clinical manifestations of patients with lumbar spinal stenosis: mediation analysis with bootstrapping. Spine J 15:238-246, 2015

13. Kim HJ, Kim SC, Kang KT, Chang BS, Lee CK, Yeom JS: Influence of educational attainment on pain intensity and disability in patients with lumbar spinal stenosis: mediation effect of pain catastrophizing. Spine (Phila Pa 1976) 39:E637-E644, 2014

14. Kovacs FM, Seco J, Royuela A, Peña A, Muriel A: The correlation between pain, catastrophizing, and disability in subacute and chronic low back pain: a study in the routine clinical practice of the Spanish National Health Service. Spine (Phila Pa 1976) 36:339-345, 2011

15. Loftus RW, Yeager MP, Clark JA, Brown JR, Abdu WA, Sengupta DK, et al: Intraoperative ketamine reduces perioperative opiate consumption in opiate-dependent patients with chronic back pain undergoing back surgery. Anesthesiology 113:639-646, 2010

16. Lohman CM, Tallroth K, Kettunen JA, Lindgren KA: Comparison of radiologic signs and clinical symptoms of spinal stenosis. Spine (Phila Pa 1976) 31:1834-1840, 2006

17. Myles PS, Weitkamp B, Jones K, Melick J, Hensen S: Validity and reliability of a postoperative quality of recovery score: the QoR-40. Br J Anaesth 84:11-15, 2000

18. Naik BI, Nemergut EC, Kazemi A, Fernández L, Cederholm SK, McMurry TL, et al: The effect of dexmedetomidine on postoperative opioid consumption and pain after major spine surgery. Anesth Analg 122:1646-1653, 2016

19. Papaioannou M, Skapinakis P, Damigos D, Mavreas V, Broumas G, Palgimesi A: The role of catastrophizing in the prediction of postoperative pain. Pain Med 10:1452-1459, 2009

20. Pavlin DJ, Sullivan MJ, Freund PR, Roesen K: Catastrophizing: a risk factor for postsurgical pain. Clin J Pain 21:83-90, 2005

21. Phillips FM, Slosar PJ, Youssef JA, Andersson G, Papatheofanis F: Lumbar spine fusion for chronic low back pain due to degenerative disc disease: a systematic review. Spine (Phila Pa 1976) 38:E409-E422, 2013

22. Picavet HS, Vlaeyen JW, Schouten JS: Pain catastrophizing and kinesiophobia: predictors of chronic low back pain. Am J Epidemiol 156:1028-1034, 2002

23. Rahim-Williams FB, Riley JL III, Herrera D, Campbell CM, Hastie BA, Fillingim RB: Ethnic identity predicts experimental pain sensitivity in African Americans and Hispanics. Pain 129:177-184, 2007

24. Rodrigues-De-Souza DP, Fernández-De-Las-Peñas C, Martín-Vallejo FJ, Blanco-Blanco JF, Moro-Gutiérrez L, Alburquerque-Sendín F: Differences in pain perception, healthrelated quality of life, disability, mood, and sleep between Brazilian and Spanish people with chronic non-specific low back pain. Braz J Phys Ther 20:412-421, 2016
25. Ruscheweyh R, Nees F, Marziniak M, Evers S, Flor H, Knecht S: Pain catastrophizing and pain-related emotions: influence of age and type of pain. Clin J Pain 27:578-586, 2011

26. Schreiber KL, Campbell C, Martel MO, Greenbaum S, Wasan AD, Borsook D, et al: Distraction analgesia in chronic pain patients: the impact of catastrophizing. Anesthesiology 121:1292-1301, 2014

27. Sirvanci M, Bhatia M, Ganiyusufoglu KA, Duran C, Tezer M, Ozturk C, et al: Degenerative lumbar spinal stenosis: correlation with Oswestry Disability Index and MR imaging. Eur Spine J 17:679-685, 2008

28. Snaith RP: The Hospital Anxiety And Depression Scale. Health Qual Life Outcomes 1:29, 2003

29. Sommer M, Geurts JW, Stessel B, Kessels AG, Peters ML, Patijn J, et al: Prevalence and predictors of postoperative pain after ear, nose, and throat surgery. Arch Otolaryngol Head Neck Surg 135:124-130, 2009

30. Sullivan MJL, Bishop SR, Pivik J: The Pain Catastrophizing scale: development and validation. Psychol Assess 7:524532, 1995

31. Sullivan MJ, D'Eon JL: Relation between catastrophizing and depression in chronic pain patients. J Abnorm Psychol 99:260-263, 1990

32. Sullivan MJ, Lynch ME, Clark AJ: Dimensions of catastrophic thinking associated with pain experience and disability in patients with neuropathic pain conditions. Pain 113:310-315, 2005

33. Sullivan MJ, Thorn B, Haythornthwaite JA, Keefe F, Martin M, Bradley LA, et al: Theoretical perspectives on the relation between catastrophizing and pain. Clin J Pain 17:52-64, 2001

34. Tripp DA, Stanish WD, Reardon G, Coady C, Sullivan MJ: Comparing postoperative pain experiences of the adolescent and adult athlete after anterior cruciate ligament surgery. J Athl Train 38:154-157, 2003

35. Turner JA, Jensen MP, Warms CA, Cardenas DD: Catastrophizing is associated with pain intensity, psychological distress, and pain-related disability among individuals with chronic pain after spinal cord injury. Pain 98:127-134, 2002

36. van Tulder MW, Ostelo R, Vlaeyen JW, Linton SJ, Morley SJ, Assendelft WJ: Behavioral treatment for chronic low back pain: a systematic review within the framework of the Cochrane Back Review Group. Spine (Phila Pa 1976) 25:26882699, 2000

\section{Disclosures}

The authors report no conflicts of interest concerning the materials or methods used in this study or the findings specified in this paper.

\section{Author Contributions}

Conception and design: Naik, Dunn, Durieux, Nemurgut. Acquisition of data: Naik, Fernández, Smith-Straesser, Jhaveri, Spanos, Thames, Spencer, Lloyd, Stuart, Ye, Bray. Analysis and interpretation of data: Naik, Durieux. Drafting the article: Naik, Dunn, Durieux. Critically revising the article: Nemurgut. Reviewed submitted version of manuscript: Naik, Dunn, Durieux, Fernández, Tsang, Smith-Straesser, Jhaveri, Spanos, Thames, Spencer, Lloyd, Stuart, Ye, Bray. Approved the final version of the manuscript on behalf of all authors: Naik. Statistical analysis: Naik, Tsang. Study supervision: Naik.

\section{Correspondence}

Bhiken I. Naik, Department of Anesthesiology, University of Virginia, Box 800710, Charlottesville, VA 22911. email: bin4n@ virginia.edu. 